\title{
Tamanho da área de vida e padrão de uso do espaço em grupos de sagüis, Callithrix jacchus (Linnaeus) (Primates, Callitrichidae)
}

\author{
Carla Soraia Soares de Castro ${ }^{1}$ \\ ${ }^{1}$ Departamento de Fisiologia, Universidade Federal do Rio Grande do Norte. Caixa postal 1511, 59078-970 Natal, Rio \\ Grande do Norte, Brasil. E-mail: carla@cb.ufrn.br

\begin{abstract}
Home range size and pattern of range use in common marmoset groups, Callithrix jacchus (Linnaeus) (Primates, Callitrichidae). The influence of abundance and distribution of fruits and gums resources in home range size and pattern of range use were investigated in Callithrix jacchus. From October 1996 to January 1998 two groups (QT and PB) that lived in a National Forest/ IBAMA-RN in northeastern Brazil (6 ${ }^{\circ} 5^{\prime} \mathrm{S}, 35^{\circ} 12^{\prime} \mathrm{W}$ ), were observed once a week by instantaneous scan sampling. The frequency of quadrant's visit in the home range was recorded at five minute intervals. Trees used for feeding on fruits and/or gum by the study groups were marked with flagging tape and numbered. Samples of the food items were collected for identification. Data on the temporal variation in fruits abundance was based on the monthly phenological observations of the marked trees. Study groups showed small home range size (QT: 2.4 ha and PB: 0.7 ha). No significant differences in home range size between dry and wet months were found, but groups showed a tendency to broadened the range use in the wet months. This revealed a behavioral strategy which marmosets exploited more gums face to decreased pattern of range use and home range size.
\end{abstract} \\ in fruits abundance. The abundance and distribution in clusters of fruits and gums resources influenced the
}

KEY WORDS. Common marmosets, Callitrhix jacchus, home range, range use.

Diferenças no tamanho da área de vida e no padrão de uso do espaço em primatas parece ser uma resposta às variáveis ambientais e sociais tais como distribuição e abundância das fontes de alimento, disponibilidade de água, locais de dormir, disponibilidade de parceiros sexuais, presença ou ausência de competidores e/ou predadores, bem como tamanho do corpo e necessidades metabólicas (MacNab 1963, Struhsaker 1974, Dawson 1979, GAUTIER-Hion et al. 1981).

O sagüi, Callithrix jacchus (Linnaeus, 1758), é um pequeno primata nativo do nordeste brasileiro, vive em grupos sociais, contendo de três a 15 animais formados por indivíduos adultos reprodutores e não reprodutores, subadultos, juvenis e infantes (STEvenson \& Rylands 1988). Cada grupo social ocupa uma determinada área denominada de área de vida. Alguns autores têm verificado que as diferenças no tamanho da área de vida e no padrão do uso do espaço, nas espécies do gênero Callithrix Erxleben, 1777, são influenciadas pela estrutura e composição florística do habitat, pela distribuição e abundância de invertebrados, frutos e árvores de goma, bem como pela presença de grupos vizinhos (Rylands 1986, SCANLON et al. 1989).

Nesse sentido, o objetivo deste estudo foi verificar a influência da abundância e da distribuição das fontes de frutos e goma no tamanho da área de vida e no uso do espaço em grupos de sagüis (Callithrix jacchus).

\section{MATERIAL E MÉTODOS}

O estudo foi realizado na Floresta Nacional (FLONA/ IBAMA), localizada no município de Nísia Floresta, no estado do Rio Grande do Norte $\left(6^{\circ} 5^{\prime} \mathrm{S}\right.$ e $\left.35^{\circ} 12^{\prime} \mathrm{W}\right)$. A região é a maior área do estado com clima subúmido, onde os meses chuvosos são de março a agosto e os meses secos de setembro a fevereiro.

A FLONA tem uma área total de 170 ha divididos em fragmentos florestais, sendo 70 ha de mata Atlântica, 50 ha de tabuleiro litorâneo e 50 ha de área de plantações, onde encontram-se eucaliptos (Eucalyptus citriodora), coco (Cocos nucifera) e espécies frutíferas. Tais locais foram demarcados utilizando-se um sistema de trilhas alfanumérico, formando uma rede de quadrantes de $25 \times 25 \mathrm{~m}$.

Dois grupos de sagüis, denominados de QT e PB, foram observados de outubro de 1996 a setembro de 1997 e de fevereiro de 1997 a janeiro de 1998, respectivamente. O grupo QT vivia na área de plantações, enquanto o grupo $\mathrm{PB}$ estava isolado do primeiro, ocupando a borda da mata Atlântica e uma área fora da FLONA denominada de coqueiral.

Tais grupos foram observados uma vez por semana através do método de varredura instantânea no qual foi registrada, a intervalos de cinco minutos, a freqüência de visitas a cada quadrante nas suas áreas de vida. Foi calculada a percentagem de 
visita aos quadrantes que foram classificados em muito $(10<$ $15 \%$ e/ou $>15 \%)$, regular $(1<5<10 \%)$ e pouco $(0<1 \%)$ visitados.

As árvores, das quais os sagüis consumiram os frutos e/ ou a goma, foram marcadas utilizando fitas com um número de identificação, sendo realizado um estudo fenológico mensal para identificar o período de abundância de frutos.

Foi utilizado o teste do Qui-quadrado para verificar se havia diferença na freqüência de visitas, pelos grupos de sagüis, aos quadrantes nos meses secos e chuvosos. Já para comparar o tamanho da área de vida nos meses secos e chuvosos foi utilizado o teste-t. Além disso, foi utilizado o índice de agregação (Ia) para descrever o padrão de distribuição das árvores com frutos e das árvores de goma. Quando $\mathrm{Ia}=1$, a distribuição é randômica; Ia $>1$, a distribuição é agregada e Ia $<1$, a distribuição é uniforme (SANTOS 1978).

\section{RESULTADOS}

Foi realizada uma estimativa da disponibilidade nas fontes de frutos e goma presentes na área de vida de cada grupo. De um total de 84 árvores utilizadas no consumo de frutos e/ ou goma, 42 estavam localizadas na área de plantações (média de seis árvores/quadrante), 33 na borda da mata Atlântica (média de 3,6 árvores/quadrante) e nove no coqueiral (média de 3,0 árvores/quadrante). A densidade de árvores variou nas áreas de vida dos grupos. $\mathrm{Na}$ área de plantações as árvores estavam distribuídas em sete quadrantes, na borda da mata Atlântica, em nove quadrantes, e no coqueiral em três quadrantes.

Foram identificadas cinco famílias contendo 10 espécies e nove gêneros de plantas que serviram como fonte de alimento para os grupos de sagüis. O grupo QT utilizou nove espécies vegetais, das quais cinco foram incluídas no consumo de frutos, três se apresentaram como fontes de goma e uma serviu como fonte de frutos e goma (Tab. I).

Já o grupo PB utilizou duas espécies vegetais presentes no coqueiral. Em uma delas consumiu frutos e goma e em uma consumiu apenas os frutos (Tab. I). Na borda da mata Atlântica esse grupo utilizou, também, três espécies, em duas espécies consumiu apenas os frutos e em uma consumiu frutos e goma (Tab. I).

As espécies vegetais, utilizadas pelo grupo QT, apresentaram frutos nos meses secos e chuvosos. Nos meses de dezembro, janeiro e fevereiro a manga (Mangifera indica L.), a serigüela (Spondias purpurea L.), a cajá-manga (Spondias dulcis Sol. ex Parkinson) e a embaúba (Cecropia adenopus Mart.) estavam em frutificação. Nos meses de abril a setembro a cajámanga (Spondias dulcis Sol. ex Parkinson), a copiúba (Tapirira guianensis Aubl.) e a goiaba (Psidium guayava L.) apresentaram frutos. Apesar da disponibilidade de frutos abranger os meses secos e chuvosos, observou-se depressões nos perfis de frutificação nos meses de outubro, novembro, março e junho (Fig. 1a).

No grupo PB, a embaúba (Cecropia adenopus Mart.) e o cumichá (Allophylus puberalis (Camb.) Radek.) apresentaram frutos nos meses de fevereiro. Já a copiúba (Tapirira guianensis Aubl.) teve seu pico de frutificação no mês de abril. A manga (Mangifera indica L.) e o caju (Anacardium occidentale L.) apresentaram frutos nos meses de novembro e dezembro (Fig. $1 \mathrm{~b})$.

Revista Brasileira de Zoologia 20 (1): 91-96, março 2003

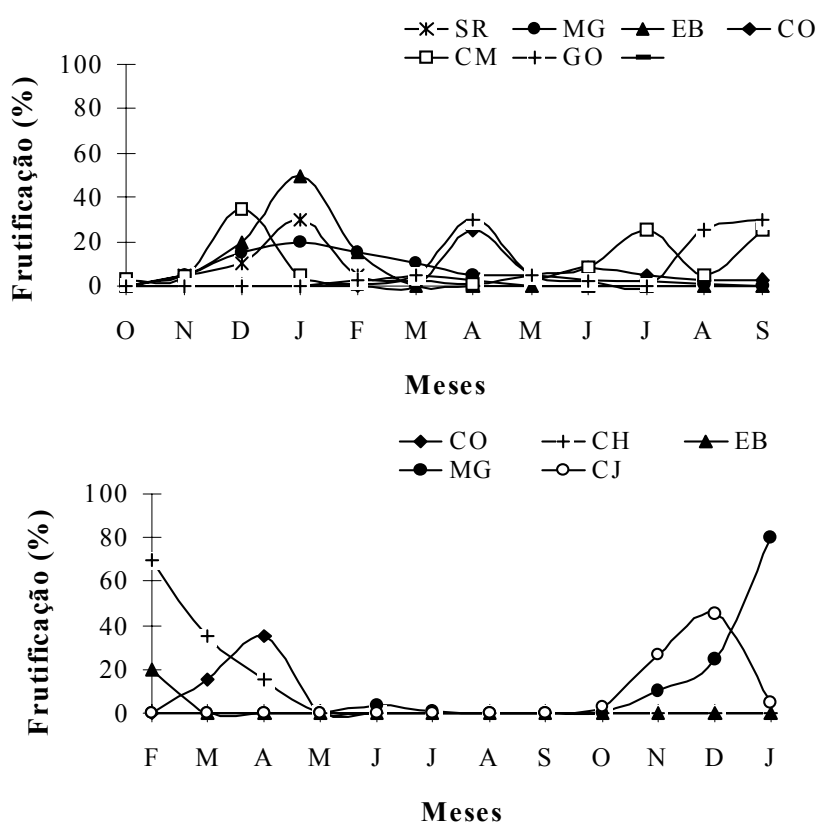

Figura 1. Perfis de frutificação das espécies vegetais utilizadas pelos grupos QT (a) e PB (b). (CJ) Caju, (MG) manga, (CO) copiúba, (SR) sirigüela, (CM) cajá-manga, (GO) goiaba, (EB) embaúba, (CH) cumichá.

Os grupos de sagüis estudados apresentaram áreas de vida de tamanho pequeno, as quais variaram, mensalmente, de 0,8 a 2,4 ha no grupo QT e de 0,3 a 1,4 ha no grupo PB. O grupo QT apresentou uma área de vida de 2,4 ha, utilizando 1,17+0,2 ha nos meses secos e $1,22+0,3$ ha nos meses chuvosos. O grupo $\mathrm{PB}$ apresentou uma área de vida menor, aproximadamente 0,7 ha, sendo utilizado $0,29+0,1$ ha da área nos meses secos e $0,40+0,2$ ha nos meses chuvosos. Embora os dados mostrem uma tendência para os grupos de sagüis ampliarem o tamanho das suas áreas de vida nos meses chuvosos, não foi encontrada diferença significativa no tamanho das áreas entre os meses secos e chuvosos (QT: $\mathrm{t}=0,43 ; \mathrm{N}=20 ; \mathrm{p}>0,05 ; \mathrm{PB}$ : $\mathrm{t}=0,24$; $\mathrm{N}=18 ; \mathrm{p}>0,05)$.

Através do índice de agregação (Ia), verificou-se que as fontes de frutos utilizadas pelos grupos QT $(\mathrm{Ia}=8,9)$ e PB $(\mathrm{Ia}=$ $2,8)$ apresentaram um padrão de distribuição agrupado, bem como as árvores de goma (QT: Ia = 8,2 e PB: Ia =2,3).

Foi observada uma relação positiva entre o período de frutificação das espécies citadas acima e a freqüência de visitas aos diversos quadrantes (QT: $X^{2}=7016,344 ; \mathrm{gl}=5$; $\mathrm{p}<0,05$; PB: $\left.X^{2}=6062,216 ; \mathrm{gl}=5 ; \mathrm{p}<0,05\right)$. Nos meses secos, os quadrantes mais visitados pelo grupo QT foram aqueles localizados a sul e a sudoeste da sua área de vida $(5<10 \%$ e $>15 \%)$. Em tais quadrantes, estavam localizadas as árvores de manga, serigüela, cajá-manga, embaúba e cupiúba. As quatro primeiras espécies foram utilizadas no consumo dos frutos, que estavam abundantes nos meses de dezembro, janeiro e fevereiro, na última os sagüis consumiram goma principalmente nos meses de outubro e novembro, período no qual ocorreu uma dimi- 
Tamanho da área de vida e padrão de uso do espaço...

Tabela I. Plantas utilizadas pelos grupos QT e PB como fontes de frutos e goma.

\begin{tabular}{|c|c|c|c|}
\hline Famílias & Nome comum & Espécie & Utilização \\
\hline \multirow[t]{5}{*}{ Anacardiaceae } & Caju & Anacardium occidentale & QT (goma) e PB (frutos e goma - coqueiral) \\
\hline & Manga & Mangifera indica & QT (frutos) e PB (frutos - coqueiral) \\
\hline & Copiúba & Tapirira guianensis & QT (frutos e goma) e PB (frutos e goma - mata Atlântica) \\
\hline & Serigüela & Spondias purpurea & QT (frutos) \\
\hline & Cajá-manga & Spondias dulcis & QT (frutos) \\
\hline Myrtaceae & Goiaba & Psidium guayara & QT (frutos) \\
\hline \multirow[t]{2}{*}{ Mimosoideae } & Timbaúba & Enterolobium contortisiliquum & QT (goma) \\
\hline & Sabiá & Mimosa caesalpiniaefolia & QT (goma) \\
\hline Cecropiaceae & Embaúba & Cecropia adenopus & QT (frutos) e PB (frutos - mata Atlântica) \\
\hline Sapindaceae & Cumichá & Allophylus puberalis & PB (frutos - mata Atlântica) \\
\hline
\end{tabular}
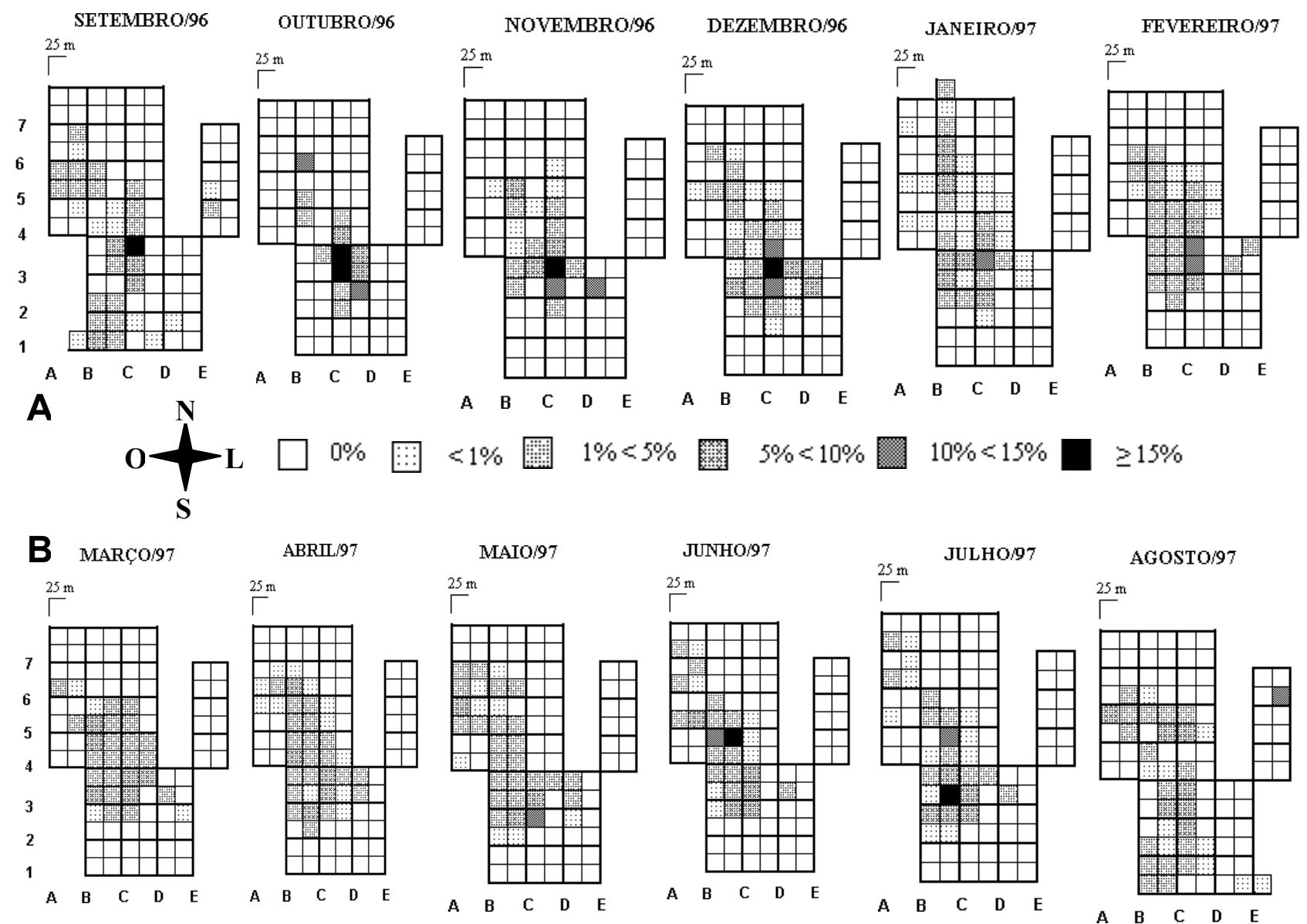

Figura 2. Tamanho da área de vida e o uso do espaço, pelo grupo QT nos meses secos - de setembro a fevereiro (A) e chuvosos - de março a agosto (B). 

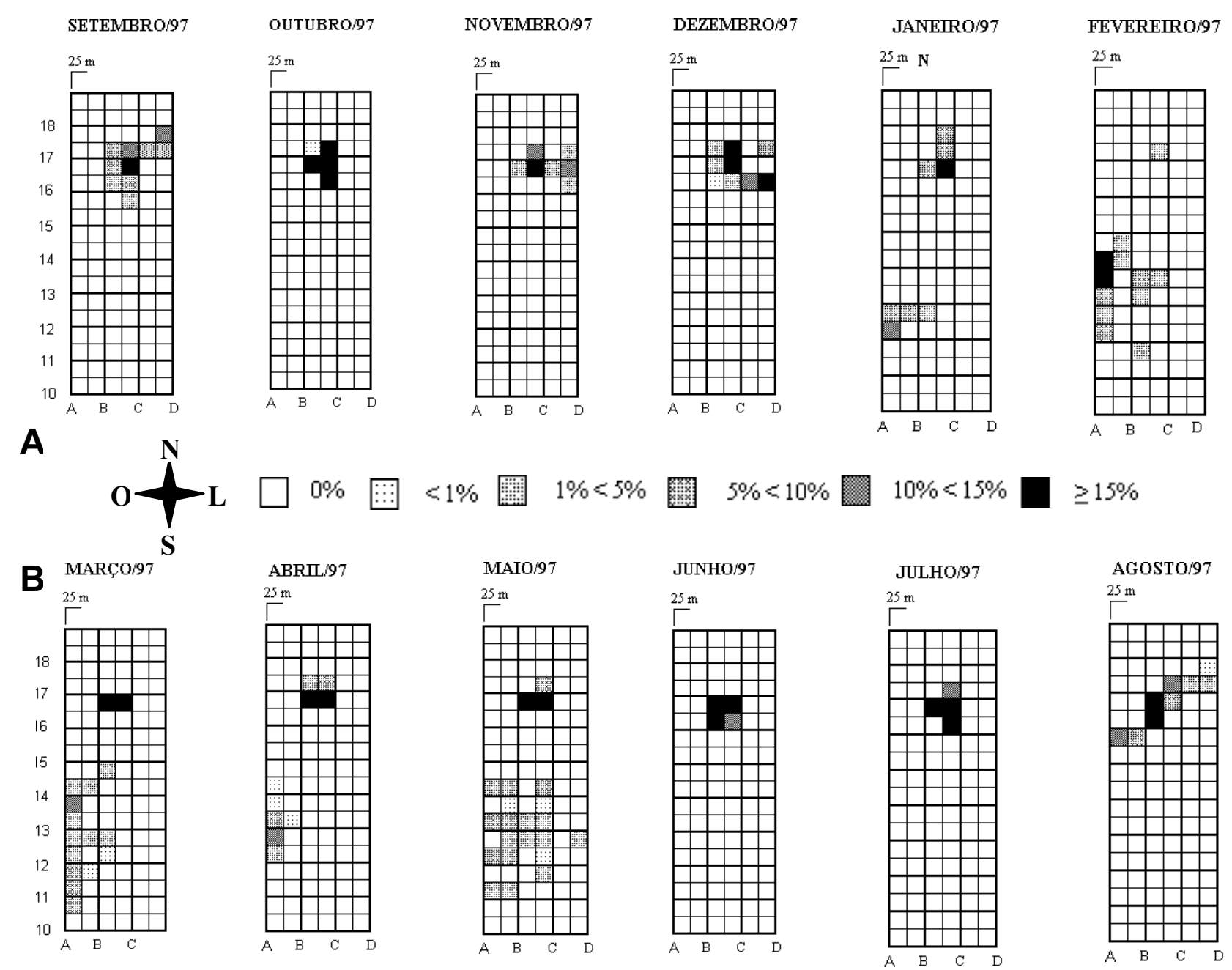

Figura 3. Tamanho da área de vida e o uso do espaço, pelo grupo PB nos meses secos - de setembro a fevereiro (a) e chuvosos - de março a agosto (b).

nuição de frutos (Fig. 2a). Nos meses chuvosos, o grupo utilizou os quadrantes onde estavam as árvores de copiúba e goiaba que apresentaram frutos de abril a agosto. Nos mês de março, quando os frutos estavam reduzidos, os sagüis se deslocaram para o fragmento de tabuleiro litorâneo, ampliando assim o uso do espaço, onde consumiram goma de algumas árvores de copiúba presentes na área (Fig. 2b).

Nos meses secos o grupo PB utilizou preferencialmente $(5<10 \%$ e $>15 \%)$ os quadrantes localizados a nordeste (coqueiral) e sudoeste (borda da mata Atlântica) da sua área de vida. Na parte nordeste, estavam localizadas as árvores de caju e manga que apresentaram frutos nos meses de novembro e dezembro. Na parte sudoeste, encontravam-se o cumichá e a embaúba que tiveram o início da frutificação em fevereiro (Fig. 3a). Nos meses chuvosos, período em que os frutos de caju e manga, estavam escassos, o grupo utilizou o caju, presente no coqueiral, na exploração de goma, mas ampliou a sua área consumindo os frutos da copiúba que estavam na borda da mata Atlântica e apresentou seu pico de frutificação em abril (Fig. 3b).

\section{DISCUSSÃO}

Alguns estudos têm demonstrado que espécies mais gomívoras, como Callithrix jacchus (0,5 ha), Callithrix penicillata E. Geoffroy, 1829 (3,5 ha) e Cebuela Gray, 1866 (0,1 ha), apresentam áreas de vida pequenas quando comparadas com espécies mais frugívoras, como Callithrix humeralifer E. Geoffroy, 1829 (28,25 ha), Callithrix kuhli Wied-Neuwied, 1826 (10-12 ha) e Callithrix aurita Humboldt, 1812 (11,5 ha), que têm áreas de vida de tamanho intermediário, enquanto que as espécies dos gêneros Saguinus Hoffmannsegg, 1807 e Leontopithecus Lesson, 1820 utilizam áreas grandes em torno de 20 a 40 ha (RyLAnds 
1986, Muskin 1986, SteVenson \& Rylands 1988, Faria 1989, Garber 1993).

Os grupos de sagüis estudados apresentaram áreas de vida pequenas. Estas estão dentro da faixa de variação descrita para o gênero Callithrix (0,5 a 35,5 ha). Como a goma é um item considerado como a base da dieta do sagüi, o pequeno tamanho das áreas de vida pode ser explicado pelo fato da goma ser um recurso que está disponível o ano inteiro, sendo suficiente poucas fontes para suprir as necessidades do grupo (Alonso \& LANGGUTH 1989). Por outro lado, os frutos apresentam marcante variação sazonal e suas fontes estão amplamente distribuídas de forma que as espécies mais frugívoras utilizam uma área maior do que as gomívoras (RYLANDS \& FARIA 1993).

O tamanho das áreas de vida dos grupos estudados não variou significativamente entre os meses secos e chuvosos. Isso se deve ao fato das fontes de frutos e goma, presentes na área da plantação, na borda da mata Atlântica e no coqueiral estarem distribuídas em pequenos agrupamentos. Os sagüis não precisaram percorrer longas distâncias para obter frutos e/ou goma, uma vez que estes se encontravam agrupados em pequenos locais das suas áreas de vida.

Em grupos de Saguinus oedipus Linnaeus, 1758, Saguinus fuscicollis Spix, 1823 e Saguinus imperator Goeldi, 1907, a localização das fontes de frutos, que estavam distribuídas em agrupamentos, minimizou as distâncias percorridas para obter alimento (Dawson 1979, Terborgh 1983).

Embora o tamanho das áreas de vida não tenha variado significativamente nos meses secos e chuvosos, observou-se que os grupos de sagüis ampliaram o uso do espaço, através das visitas a um número maior de quadrantes, nos meses chuvosos. Isso teve uma estreita relação com a variação na abundância de frutos, uma vez que quando algumas fontes estavam escassas os grupos passaram a explorar mais ativamente as fontes de goma (CASTRO et al. 2000).

Resultado semelhante foi obtido com um grupo de Callithrix geoffroyi Humboldt, 1812, pois este reduziu o uso da sua área de vida nos meses de dezembro e janeiro, período em que se concentrou a frutificação das espécies utilizadas como alimento. Durante os outros meses o grupo utilizou um espaço maior devido à necessidade, frente à escassez de frutos, de utilizar um maior número de árvores de goma (PASSAMANI \& RYLANDS 2000).

Diferenças na estrutura e composição florística do habitat podem influenciar o tamanho da área de vida e o uso do espaço em algumas espécies do gênero Callithrix (RYLANDs 1986). O grupo PB apresentou uma área de vida de tamanho muito inferior $(0,7 \mathrm{ha})$ a do grupo QT $(2,4 \mathrm{ha})$. Tal diferença pode ter relação com o tipo de vegetação presente no local que cada um ocupou. A área de plantação, onde o grupo QT vivia, continha um maior número de espécies frutíferas, com sobreposição nos períodos de frutificação, enquanto que no coqueiral e na borda da mata, áreas utilizadas pelo grupo $\mathrm{PB}$, o número de espécies utilizadas foi mais reduzido e a frutificação se concentrou nos meses de fevereiro, março, dezembro e janeiro (CASTRO et al. 2000).

\section{CONCLUSÕES}

A distribuição, em pequenos agrupamentos, das fontes de goma e frutos, bem como a abundância desta última, influenciaram o padrão de uso do espaço e o tamanho das áreas de vida dos grupos de sagüis estudados. Considerando que a área do estudo sofreu uma grande pressão antrópica levando a sua fragmentação, os resultados desse estudo demonstram a plasticidade comportamental do Callithrix jacchus, através da estratégia de exploração das fontes de goma frente à escassez de frutos, permitindo a essa espécie utilizar os mais diversos ambientes, podendo ser encontrada desde fragmentos florestais até a caatinga nordestina.

\section{AGRADECIMENTOS}

Ao Instituto Brasileiro do Meio Ambiente e Recursos Naturais Renováveis (IBAMA-RN) por permitir a coleta de dados na área da Floresta Nacional, ao CNPq pelo suporte financeiro, a bióloga Maria do Socorro Borges Freire pelo levantamento botânico da área do estudo e a Profa. Iracema Loyola por acrescentar os autores na lista das espécies vegetais.

\section{REFERÊNCIAS BIBLIOGRÁFICAS}

Alonso, C. \& A. Langguth. 1989. Ecologia e comportamento de Callithix jacchus (Primates: Callitrichidae) numa ilha de floresta Atlântica. Revista Nordestina de Biologia, Recife, 6 (2): 105-137.

Castro, C.S.S; A. Araújo; C.R. Alho \& M.M. Dias-Filho. 2000. Influência da distribuição e disponibilidade dos frutos, na dieta e no uso do espaço em sagüis-do-nordeste (Callithrix jacchus), p. 65-80. In: C. Alonso \& A. Langguth (Eds). A Primatologia no Brasil. 7. João Pessoa, SBPr, Ed. Universitária, 220p.

Dawson, G.A. 1979. The use of time and space by the Panamanian tamarin, Saguinus oedipus. Folia Primatologica, Switzerland, 31: 253-284.

FARIA, D.S. 1989. O estudo de campo com o mico estrela no Planalto Central brasileiro, p. 109-121. In: C. Ades (Ed). Etologia de animais e de homens. São Paulo, EDUSP, 300p.

GARBER, P.A. 1993. Feeding ecology and behaviour of the genus Saguinus, p. 273-295. In: A.B. Rylands (Ed). Marmosets and Tamarins (Systematics, Behaviour, and Ecology). New York, Oxford Univ. Press, 396p.

Gautier-Hion, A.; J.P. Gautier \& R. QuRis. 1981. Forest structure and fruit availability as complementary factors influencing habitat use by a troop of monkeys (Cercopithecus cephus). Terre Vie, Paris, 35: 511-536.

MACNNAB, B.K. 1963. Bioenergetics and the determination of home range size. The American Naturalist 97: 133-140.

Muskin, A. 1986. Preliminary field observations of Callithrix aurita (Callitrichidae, Cebinae), p. 79-82. In: M.T. Mello (Ed). A Primatologia no Brasil. 2. Brasília, SBPr, 232p.

Passamani, M. \& A.B. Rylands. 2000. Home range of a Geoffroy's marmoset group, Callithrix geoffroyi (Primates, Callithrichidae) in South-eastern Brazil. Revista Brasileira de Biologia 60 (2): 1-10.

RYLANDS, A.B. 1986. Ranging behavior and habitat preference of a wild marmoset group, Callithrix humeralifer (Callitrichidae-Primates). Journal Zoological of London 210: 126.

RYlands, A.B. \& D.S. FaRia. 1993. Habitats, feeding, and home range size in the genus Callithrix, p. 262-272. In: A.B. RyLANDS (Ed). Marmosets and Tamarins (Systematics, Behaviour, and Ecology). New York, Oxford Univ. Press, 396p.

SANTOS, E.P. 1977. Dinâmica de populações aplicada à pesca 
e psicultura: distribuição espacial. São Paulo, EDUSP, 129p. SCAnlon, C.E.; N.R. Chalmers \& M.A.O. Monteiro da Cruz. 1989. Changes in the size, composition and reproductive condition of wild marmoset groups (Callithrix jacchus jacchus) in northeast Brazil. Primates 29: 295-305.

Stevenson, M.F. \& A.B. Rylands. 1988. The marmosets, genus Callithrix, p.131-211. In: R.A. MitTermeier; A.B. Rylands; A.F. CoImbra-Filho \& G.A.B. Fonseca (Eds). Ecology and Behavior of Neotropical Primates. Washington, D.C., World Wildlife Fund, 610p.

STRUHSAKER, T.T. 1974. Correlates of ranging behaviour in a group of red colobus monkeys, Colobus badius tephrosceles. The American Zoologist 14: 177-184.

Terborg, J. 1983. Five New World Primates: A Study in Comparative Ecology. Princeton, Princeton Univ. Press, 304p.

Recebido em 01.VIII.2002; aceito em 28.II.2003.

Revista Brasileira de Zoologia 20 (1): 91-96, março 2003 